\title{
Future Developments in Low Temperature Detectors for CMB and Submm Astronomy
}

\author{
Sunil Golwala \\ Division of Physics, Mathematics, and Astronomy, California Institute of Technology, Pasadena, CA 91125
}

\begin{abstract}
We summarize the wide range of current and upcoming developments in low temperature detectors for CMB and submillimeter astronomy. We discuss work in sensor development, photon coupling and filtering architectures, and polarimetry and how these tie to applications requirements.
\end{abstract}

Keywords: astronomical instrumentation, cosmic microwave background, submillimeter astronomy, far-infrared astronomy, dust polarimetry, cosmic microwave background polarimetry

PACS: $95.55 . \mathrm{Cs}, 95.55 . \mathrm{Jz}, 95.55 . \mathrm{Qf}$

\section{INTRODUCTION}

There has been an explosion in the development of lowtemperature detectors for cosmic microwave background studies and submillimeter astronomy in the last two decades. The impetus was provided by the first detections of cosmic microwave background (CMB) anisotropy by COBE/DMR [1], of the cosmic far-infrared background (CFIRB) by COBE/DIRBE and COBE/FIRAS [2, 3, $4,5]$, and of optically obscured submillimeter galaxies by SCUBA [6, 7], all in the early to mid 1990 s. They provided the first examples of significant cosmology and extragalactic astronomy done using the far-infrared, submm, and $\mathrm{mm}$ bands, leading eventually to the recently launched Planck and Herschel satellite missions. Figure 1 shows the brightness of the infrared and microwave backgrounds: together, the bulk of the photon energy in the universe lies in these bands.

It was immediately clear that better detector sensitivities and larger pixel counts, combined with innovative optical system designs, would be required to fully exploit these newly important spectral bands. Low-temperature detectors are central to these developments because they provide background-limited performance across the entire spectral range of interest here, from $90 \mathrm{GHz}(\sim 3$ $\mathrm{mm})$ to $5 \mathrm{THz}(60 \mu \mathrm{m})$. The focus of this paper is current and upcoming work in these areas, resulting in large focal-plane arrays for ground-, balloon-, and eventually space-borne imaging, polarimetry, and spectroscopy.

\section{IMAGING, SPECTROSCOPY, AND POLARIMETRY}

Figure 1 shows that most photons in the FIR/submm/mm bands come from either the $\mathrm{CMB}$ or thermal emission from dust that has been heated by photons from stars or

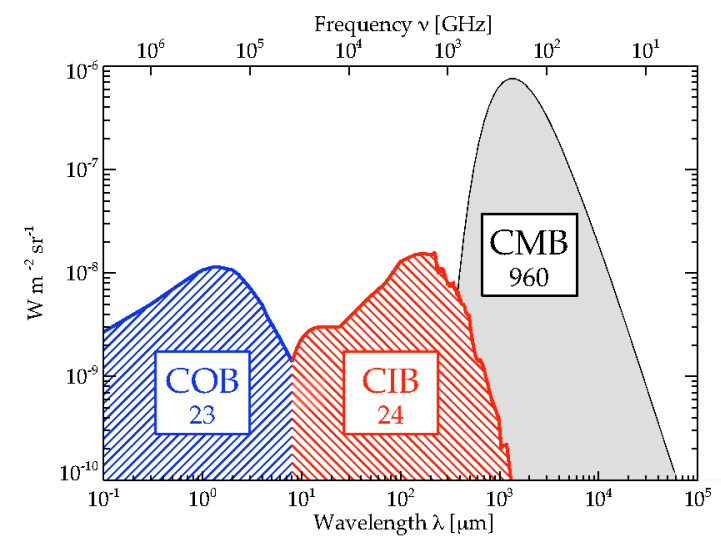

FIGURE 1. The logarithmic brightness spectrum of the cosmic optical, infrared, and microwave background radiations, $v B_{v}$. The numbers indicate the integrated brightness in each background in $\mathrm{nW} / \mathrm{m}^{2} / \mathrm{ster}$. Figure from [8].

energetic accretion ("CIB"). In some energetic sources, such as the galactic center, non-thermal synchrotron and free-free emission may contribute at the longer wavelengths. Spectral line emission can also be a significant component in some circumstances. In galaxy clusters, there is emission due to the Sunyaev-Zeldovich effect, the Compton scattering of CMB photons by free electrons in the intracluster plasma. Clearly, broadband imaging, with a wide range of resolution varying from an arcsecond to a degree, is the first goal.

The next task depends on the science topic. For studying how galaxies, stars, and planetary systems form and evolve, continuum imaging is only the beginning. Dust is typically a small fraction of the total baryonic mass; molecular and atomic gas dominates. The gas cools primarily via emission in spectral lines, eventually clumping to form stars and planetary systems. Line widths and 
strengths probe temperature, density, and chemical composition of the gas. Thus, one requires spectroscopy at a range of spectral resolving powers to find lines and study their detailed structure. In addition, percent-level polarimetry of dust thermal emission and spectroscopy of ion species probe the magnetic fields that are thought to play an important role in star formation.

The CMB presents a different challenge. Because its astrophysics is relatively simple, theory predicts its properties to exquisite precision, making the $\mathrm{CMB}$ a precise probe of cosmological parameters and early-universe physics. Thus, we must measure its anisotropy, polarization, and spectral shape to commensurate precision. Large arrays of background-limited detectors observing through carefully designed, well-characterized optical systems meet these requirements.

We thus divide our discussion among: detector sensitivity, large detector arrays, and "low-temperature" optical components that enable imaging, spectroscopy, and polarimetry. We refer the reader to K. Irwin's review article on multiplexing techniques for a complementary discussion of readout of large detector arrays [9]. We focus on wavelengths between roughly $60 \mu \mathrm{m}$ and $3 \mathrm{~mm}$ because sub-Kelvin low-temperature detectors are the most sensitive for this range: the quanta of low temperature detectors - phonons and quasiparticles - are much smaller than the photon energy. At longer wavelengths, coherent amplifier receivers operating at a few Kelvin and above are generally in use, while, at shorter wavelengths, the higher photon energy makes use of electronhole quanta in semiconductors at higher temperatures sufficiently sensitive. There are of course applications that can benefit from low-temperature detectors at shorter wavelengths, discussed elsewhere in this volume.

\section{DETECTOR SENSITIVITY}

Noise equivalent power (NEP) is the measure of individual detector sensitivity. The incoherent detectors that we focus on here sense total power (rather than the electric field) and are subject to a quiescent optical power (load). This "background" power results in a fundamental limit to the NEP, the so-called "background-limited infrared photometer" (BLIP) limit, due to fluctuations on the incoming power due to the discreteness of photons (shot noise and Bose (also known as wave or clumping) noise) and has the approximate form

$$
\mathrm{NEP}_{\mathrm{BLIP}}^{2}=2 h v Q+\frac{Q^{2}}{\Delta v}
$$

where the detector receives a quiescent optical load $Q$ in a spectral bandwidth $\Delta v$ centered about a frequency $v$. The goal is to develop detectors whose intrinsic noise is below the BLIP limit. The limit depends on the observing frequency, the total optical load, and the spectral bandwidth. The optical load depends on the observing site/platform and on the ambient temperature and optical quality of the telescope, while the spectral bandwidth depends on the frequency and on the mode of operation - imaging vs. spectroscopy. Thus, the BLIP limit varies with the application, presenting varying requirements on detector sensitivity.

Our generic low temperature detector consists of an optical absorber that is linked to a thermal bath via a conductance $G$ and whose temperature is monitored in some fashion. The temperature of the absorber measures the incoming optical power via $\Delta T=\Delta P / G$. Detector NEP is fundamentally limited by the conductance $G$

$$
\mathrm{NEP}_{\mathrm{G}}^{2}=4 \gamma k T_{b}^{2} G
$$

One typically encounters the limit stated in this fashion for bolometers. It arises because the quiescent power (optical and electrical) flow from the detector to the bath suffers fluctuations due to the discrete nature of the phonons that carry that power, causing temperature fluctuations indistinguishable from variations in optical power. A relation of this form holds more generally, even non-thermal detectors such as SIS tunnel junctions and kinetic inductance detectors, because they too have a link to a thermal bath (via quasiparticle decay) through which power flows via discretized carriers.

Thus, improving detector NEP requires reducing $G$. However, $G$, along with the heat capacity of the absorber and thermometer $C$, also determines the time for the detector to return to its quiescent temperature after an optical power input. Thus, practical considerations require that reducing $G$ be paired with other advances that keep this detector time constant small enough to be useful.

Figure 2 shows the steady progress in detector NEP over the past decades. The BLIP limit for various observing platforms is also indicated. The BLIP limit for continuum imaging from the ground in the FIR/submm $/ \mathrm{mm}$, roughly $3 \times 10^{-17} \mathrm{~W} / \sqrt{\mathrm{Hz}}$, was met in the mid-1990s by leg-isolated spiderweb bolometers using semiconducting neutron-transmutation-doped (NTD) germanium thermistors $[11,12]$; see Figure 3. A low-fill factor, thin mesh absorber ( $\sim 1 \mu \mathrm{m}$ thick) coated with gold provides low heat capacity. It is connected to the bath by silicon nitride legs that provide low conductance bath ( $G \sim 100-200 \mathrm{pW} / \mathrm{K}$ initially) yet are mechanically robust. Spiderweb bolometers also demonstrated BLIP performance in the lower loading conditions on balloons (BOOMERanG [13] and, later, ARCHEOPS [14], requiring $G<100 \mathrm{pW} / \mathrm{K}$ ). This, along with lab demonstrations BLIP sensitivity under space conditions, led to their selection for the Planck and Herschel missions. Planck/HFI uses a set of 52 individual spider- 


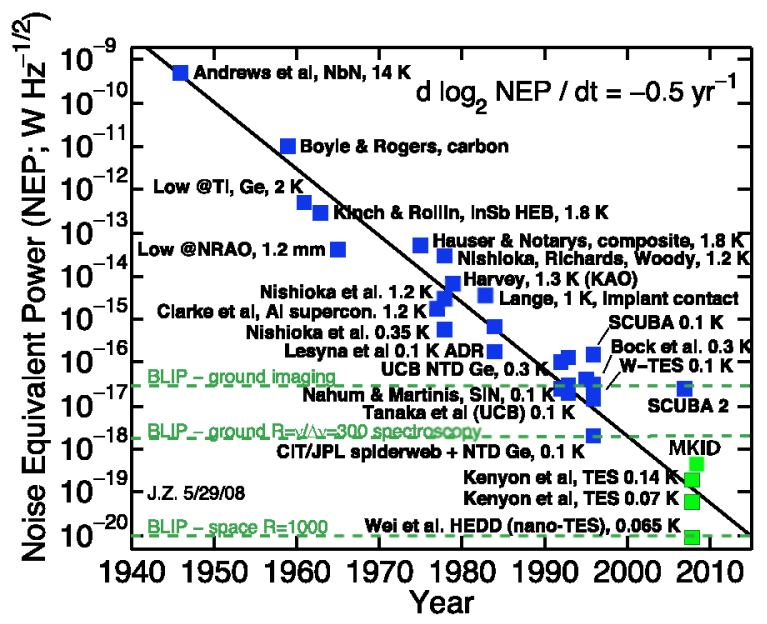

FIGURE 2. Bolometer NEP as a function of time. BLIP NEPs for various platforms and resolving powers are indicated. Blue indicates deployed receivers, green indicates lab demonstrations of electrical NEP. Figure courtesy of J. Zmuidzinas $[10]$.

web bolometers from $350 \mu \mathrm{m}$ to $3 \mathrm{~mm}$ [15], while Herschel/SPIRE uses spiderweb bolometer arrays in an imager $(139,88$, and 43 pixels at 250,360 , and $520 \mu \mathrm{m})$ and a Fourier transform spectrometer (37 and 19 pixels for 194-324 $\mu \mathrm{m}$ and 316-672 $\mu \mathrm{m}$, respectively) [16]. Both instruments employ telescopes passively cooled to $50 \mathrm{~K}$ and $80 \mathrm{~K}$, respectively. The detectors were developed by the JPL/Caltech group. SHARC-II [17] has demonstrated BLIP performance at $350 \mu \mathrm{m}$ using ion-implanted silicon thermistors on leg-isolated monolithic pop-up absorbers developed by GSFC; see Figure 4 . They have higher heat capacity than the spiderweb design, but the higher optical load from the atmosphere in this band requires higher conductances, so the time constants remain reasonable (a few msec). Herschel/PACS $(60 \mu \mathrm{m}$ to $210 \mu \mathrm{m})$ uses a hybrid design developed at CEA, employing ion-implanted silicon thermistors on mesh absorbers with leg isolation to obtain both the low conductance required for space loading and the low heat capacity needed for a reasonable time constant [18]. These technologies have produced cutting-edge science - the first high signal-tonoise maps of $\mathrm{CMB}$ anistropy indicating the universe is flat (BOOMERanG [19], MAXIMA [20]), the best CMB anistropy measurements on scales larger than a degree prior to WMAP (ARCHEOPS [21]), high precision measurements of the few-arcminute-scale anisotropy of the CMB (ACBAR [22]), SED measurements of many highredshift submillimeter galaxies (SHARC-II, e.g. [23, 24]), and the resolution of the CFIRB at the submillimeter wavelenths where it is most significant (BLAST [25]) - and promise even more via Planck and Herschel.
A number of new technologies have recently been developed with comparable sensitivity but more promise for reaching large pixel counts. Absorber-coupled transition-edge sensors (TESs) [27, 28, 29] have demonstrated background-limited performance for ground-based imaging in the APEX-SZ [30], SPT, and ACT [31] receivers. The first two employ spiderweb designs by the Berkeley group while the latter uses GSFC-manufactured pop-up absorbers with TESs [32]. TES electrothermal feedback speeds up the pop-up detectors enough for the high heat capacity of the monolithic absorber to be acceptable.

Many large-scale array designs use transmissionline-coupled bolometers. BICEP2/Keck Array and SPIDER will employ leg-isolated TESs coupled to phasedarray antennas (see following section) developed at JPL/Caltech [33]. They have demonstrated $G \sim 10 \mathrm{pW} / \mathrm{K}$ at $450 \mathrm{mK}$ for SPIDER, which will provide a NEP of $1 \times 10^{-17} \mathrm{~W} / \sqrt{\mathrm{Hz}}$ for this particularly low-loading balloon receiver. A higher conductance design, with $G=$ $70-100 \mathrm{pW} / \mathrm{K}$ at $450 \mathrm{mK}$, will meet the ground-based BLIP NEP for BICEP2/Keck Array. Berkeley has developed TESs with similar performance for their lens+dualslot dipole antenna design. NIST, CU-Boulder, Princeton, and University of Chicago are developing similar detectors that couple to planar OMT waveguide probes and have demonstrated $G=70 \mathrm{pW} / \mathrm{K}$ at $550 \mathrm{mK}$ and NEP of $2-3 \times 10^{-17} \mathrm{~W} / \sqrt{\mathrm{Hz}}$ [34]. GSFC is also developing a transmission-line-coupled design [35].

Microwave kinetic inductance detectors (MKIDs) [36] are advancing quickly and will be first deployed for astronomical observations in MKIDCam [37] and NIKA [38]. They have yielded dark NEPs below $10^{-18} \mathrm{~W} / \sqrt{\mathrm{Hz}}$. At effective conductances large enough for ground-based imaging, they approach the BLIP limit [39]. MKIDs are described in detail elsewhere in this volume [40]. A French group is developing $\mathrm{NbSi}$ detectors, varying the stoichiometry to obtain semiconducting or superconducting (TES) behavior. Their semiconducting designs promise $10^{-17} \mathrm{~W} / \sqrt{\mathrm{Hz}}$ [41] and will be deployed on a camera for the IRAM $30-\mathrm{m}$ telescope, while their superconducting designs have demonstrated $2 \times 10^{-16} \mathrm{~W} / \sqrt{\mathrm{Hz}}$ [42] and will be used on OLIMPO.

By the late 1990s, the performance of leg-isolated bolometers using semiconducting thermistors had reached the BLIP limit for ground-based low-resolution dispersive spectroscopy, roughly $2 \times 10^{-18} \mathrm{~W} / \sqrt{\mathrm{Hz}}$ and requiring $G \sim 15 \mathrm{pW} / \mathrm{K}$ at $100 \mathrm{mK}$. This limit is more stringent than for imaging or FTS because the optical load per detector is reduced from the continuum level by the spectrometer resolving power $R$. Reaching this milestone led to the Z-Spec [43] and ZEUS [44] instruments: $Z$-Spec uses 160 detectors to do $R=200$ spectroscopy over the entire 1-1.4 $\mathrm{mm}$ band simultaneously, while 

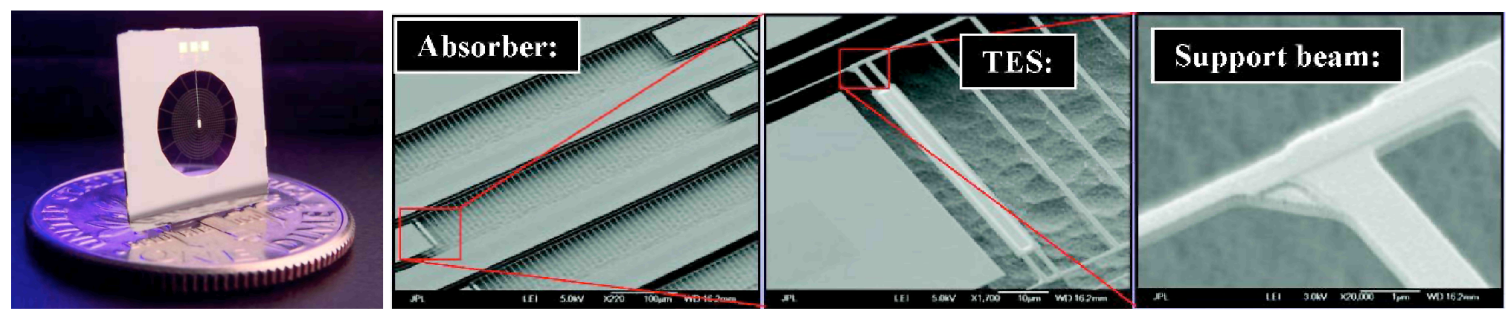

FIGURE 3. Absorber-coupled spiderweb-style detectors. Left: a spiderweb bolometer, $G \sim 100 \mathrm{pW} / \mathrm{K}$. Right three pictures: A low-conductance $(17 \mathrm{fW} / \mathrm{K}$ at $70 \mathrm{mK}$ ) leg-isolated Mo/Au TES bolometer appropriate for cooled-aperture dispersive spectroscopy in space. The thermal-isolation legs are $1 \mathrm{~mm}$ long and $0.25 \mu \mathrm{m}$ by $0.4 \mu \mathrm{m}$ in cross section. Figure courtesy of M. Kenyon [26].

ZEUS uses 32 detectors to do $R=1000$ spectroscopy over tunable portions of the 350 and $450 \mu \mathrm{m}$ windows. They have begun to do impressive science, with Z-Spec detecting the $\mathrm{CO}$ ladder in the Cloverleaf quasar, a slew of molecules in nearby galaxies $[45,46]$, and ZEUS detecting ${ }^{13} \mathrm{CO} J=6 \rightarrow 5$ in $\mathrm{NGC} 253$ [47] and ionized carbon [CII] in MIPSJ142824, a starburst galaxy at $z=1.32$ [48]. Both instruments use the $10.4-\mathrm{m}$ Caltech Submillimeter Observatory. Even more exciting is the prospect of using instruments like these on much larger apertures, such as the 50-m Large Millimeter Telescope [49] and the 25-m Cornell-Caltech Atacama Telescope [50].

The next major goal is to obtain BLIP performance for spectroscopy on cooled-aperture $(\sim 4 \mathrm{~K})$ space platforms such as SPICA (3 m) [51] and, eventually, the 6$\mathrm{m}$ SAFIR [52]/CALISTO [53]. On these platforms, optical loading is further reduced by the absence of atmosphere and the cooled optical system, thereby reducing the NEP BLIP. Dispersive spectroscopy with resolution comparable to that of Z-Spec or ZEUS requires a NEP of $5 \times 10^{-20} \mathrm{~W} / \sqrt{\mathrm{Hz}}$. Leg-isolated TES bolometers employing extreme leg geometries have yielded the very low conductances required while using electrothermal feedback to maintain usable response times. Using a $\mathrm{Mo} / \mathrm{Au}$ bilayer TES, the JPL/Caltech group has inferred a NEP of $6 \times 10^{-20} \mathrm{~W} / \sqrt{\mathrm{Hz}}$ at $T_{c}=70 \mathrm{mK}$ based on a measured leg conductance at $17 \mathrm{fW} / \mathrm{K}$ and has measured an effective time constant of $400 \mathrm{~ms}$ [26]; see Figure 3. Full integration of the geometry, TES, and readout have not yet been achieved, but should come soon. Another approach is to use very small volume TESs, in which case electron-phonon decoupling provides the low conductance link. The "hot-electron direct detectors" (HEDD) have demonstrated conductances that correspond to phonon-noise NEPs of $<10^{-18} \mathrm{~W} / \sqrt{\mathrm{Hz}}$ for $T<0.3 \mathrm{~K}$ and $<10^{-20} \mathrm{~W} / \sqrt{\mathrm{Hz}}$ for $T<0.06 \mathrm{~K}$, while an electrical NEP of $6.7 \times 10^{-18} \mathrm{~W} / \sqrt{\mathrm{Hz}}$ has been demonstrated at $T=0.324 \mathrm{~K}$ [54]. They are, quite fast due to their low heat capacity, with a calculated thermal time constant of $1.9 \mu \mathrm{s}$. A RF-multiplexing scheme is under development for them [55]. GSFC are also pursuing de- vices of this type [56, 57]. Finally, MKIDs promise performance of $10^{-20} \mathrm{~W} / \sqrt{\mathrm{Hz}}$ as long as two-level-system noise shows no amplitude component.

Given that detectors capable of doing BLIP-limited dispersive spectroscopy are not yet ready for deployment, an alternative under consideration is mating a FTS to a cooled aperture, which requires NEPs an order of magnitude less demanding, $5 \times 10^{-19} \mathrm{~W} / \sqrt{\mathrm{Hz}}$, but would nevertheless provide substantial gains over Herschel/PACS. There is a European proposal, SAFARI, for such an instrument, with both leg-isolated TESs [58] and MKIDs [59, 60, 61] under consideration.

\section{LARGE DETECTOR ARRAYS}

With BLIP performance, even for a cooled aperature in space, already in hand for imaging applications and within reach for spectroscopy, the next path to increased scientific capability is large arrays. In imaging, arrays provide a linear increase in mapping speed with pixel count. For spectroscopy, arrays can provide instantaneous coverage of large fractional bandwidths (30-50\%), multiple bands simultaneously, and/or full spectral coverage at higher resolution. The first two options can provide linear improvements in spectroscopic mapping speed in searches for known lines in objects at unknown redshifts and for line surveys.

The traditional methodology has been to have single detectors behind individually manufactured corrugated feedhorns. Because such feeds preserve polarization, absorbers metallized so as to absorb one polarization provide polarization sensitivity [62]. However, it is too costly and massive to extend this technique to arrays of more than tens of pixels. Many groups are taking a wide variety of approaches to detector arrays: single detectors and monolithic arrays behind feedhorn arrays, bare-absorber arrays, lens+antenna-coupled arrays, and phased-array antenna-coupled arrays. The techniques vary in the ways they define the beam and the beam-forming element couples light to the detector. 
The first move away from the single-feedhorn approach has been the use of monolithic detector arrays behind conical smooth-walled feedhorn arrays [63]. The feeds are typically $f \lambda$ to $2 f \lambda$ in size to maximize the use of focal plane area. The reduced horn diameter results in poorer control of beams and stray light. They have so far only been used in conjuction with cold aperture stops to ensure well-controlled primary illumination and to terminate the sidelobes of these less-than-ideal horns. Bolocam [64], MAMBO [65], LABOCA [66], APEXSZ [30], SPT, BLAST [67], and Herschel/SPIRE [16] have employed this approach to good success. All use arrays of leg-isolated absorber-coupled bolometers, in which the bolometer body is also the optical radiation absorbing element and sits behind the horn, usually in an integrating cavity. This approach is clearly challenging past the 1000-pixel level since such horns are macroscopic objects that must be machined or electroformed. Additionally, cylindrical feedhorns do not preserve polarization well and thus a polarization analyzer must precede the array. EBEX uses wire grid polarizers in front of conical smooth-walled feedhorn arrays for CMB polarization anisotropy measurements [68].

Two collaborations, one consisting of NIST, CUBoulder, Princeton, and University of Chicago and the other led by GSFC, are developing arrays of corrugated feedhorns $[69,35]$ using a platelet method [70]. Multiple sheets of silicon or metal are machined with hole patterns corrsponding to a cross section through an array of corrugated feedhorns. The various layers are aligned and permanently attached to each other using diffusion bonding (metals) or epoxy (silicon). There is not yet much data on the performance of these feeds at $\mathrm{submm} / \mathrm{mm}$ wavelengths, but it will be forthcoming soon. An Argonne National Lab/University of Chicago group are developing polarization-sensitive absorber-coupled detectors to mate to these corrugated feedhorn arrays [71].

In the last decade, various groups have demonstrated that, for frequencies appreciably below the superconducting pair-breaking energy of niobium $(720 \mathrm{GHz})$, one can construct low-loss transmission line using niobium and silicon oxide or dioxide [72]. This makes it possible to use the signal-routing techniques of microwave engineering. One can terminate a corrugated feed with a matched-impedance probe that feeds a superconducting transmission line terminating in a detector. This approach brings a number of benefits. First, by decoupling the optical system from the detector, one can make much smaller, and hence lower- $C$, detectors. This makes it possible to reduce $G$ and thus detector noise without paying a price in time constant. Second, one can pick off the two polarizations very cleanly without any consideration for detector absorber geometry, thereby reducing possible cross-polarization. Third, one can insert bandpass filters between the antenna and the detectors. The
JPL/Caltech group has demonstrated bandpass filters that are essentially 3-pole lumped-element filters using either a spiral inductor [73] or a length of transmission line [74] as the inductive element. Other groups [75, 76, 77, 78] use sequences of $\lambda / 4$ transmission line stubs.

Both groups developing corrugated feedhorn arrays are thus also developing detector arrays that incorporate antenna-coupled designs $[35,79,78,80]$. They use planar orthomode transducers (OMTs) to precisely extract the two polarizations from the feeds, and they feed the light to leg-isolated TES bolometers. The $\mathrm{C}_{\ell}$ OVER experiment had planned to use individually fabricated feedhorns but with the same kind of planar OMT/transmission line/TES bolometer design for its 150 and $220 \mathrm{GHz}$ bands. For its $90-\mathrm{GHz}$ band, $\mathrm{C}_{\ell}$ OVER intended to use a conventional waveguide OMT but then transition into finline waveguide probes to feed TES bolometers via superconducting transmission lines [81].

The natural next step is to employ the techniques of planar and phased-array antennas to eliminate feedhorns completely. Such designs derive from the superconducting twin-slot dipole antenna and its dual-polarization version developed for SIS receivers in the 1990s [82, 83], which uses a hyperhemispherical lens to focus the forward beam and to match the incoming wavefront from the telescope. The lenses are approximately $2 f \lambda$ in diameter. The antenna has a bandwidth of about $40 \%$ [83]. The lenses must be antireflection-coated and mechanically attached to the wafer on which the antennas, filters, and detectors are fabricated. Berkeley and collaborators are using the dual-slot design for the POLARBeaR CMB polarization instrument for bands at 90, 150, and $220 \mathrm{GHZ}$ to feed leg-isolated TES bolometers [75]. More recently, they have developed a sinuous antenna design that provides a much larger instantaneous bandwidth, 3:1 [77]. For both designs, filters on the transmission line exiting the antenna use a serious of $\lambda / 4$ stubs to define bandpasses.

JPL/Caltech have developed antennas with each pixel consisting of a phased array of slot dipole antennas synthesizing a forward beam without a lens. They have demonstrated dual-polarization designs with $25 \%$ bandwidth [84] and single-polarization designs with an octave of bandwidth [74]. The dual-polarization pixels are sized to $2 f \lambda$ while the wide-bandwidth, single-polarization pixels are usually sized to $2 f \lambda$ in their highest frequency band. Both designs employ lumped-element in-line filters $[73,74]$.

By contrast, a number of architectures take an "optical" as opposed to "microwave" approach. SHARCII, ACT, GISMO, MUSTANG, SCUBA-2, and Herschel/PACS all employ filled, bare-absorber arrays. Such arrays have no intrinsic beam or stray-light control, but careful optical design and baffling can compensate. They offer maximally efficient use of focal plane area by al- 
lowing no photon to go to waste. Two architectures have been developed - the pop-up design [85] (SHARCII [17] and ACT [31]), in which silicon legs attached to a linear array of detectors are bent beneath the array to closely pack multiple linear arrays to form a larger array; and waffle-pattern planar designs (GISMO [86], MUSTANG [87], SCUBA-2 [88], Herschel/PACS [89]) in which the isolating legs are in the plane of the wafer and attach to a waffle pattern of silicon that is the thermal bath. Single kilopixel arrays of both kinds have been produced, with larger focal planes obtained by assembling multiple kilopixel units. See Figure 4.

Progress in array size is summarized in Figure 5. Long-term growth in array size is most easily facilitiated by designs that are fully photolithographic and require no later assembly steps (aside from full-wafer hybridization with readout). The phased-array pixel and waffleabsorber designs satisfy these needs. However, it must also be emphasized that, for CMB experiments, control of systematics is likely to trump pixel count somewhere in the kilopixel to 10 kilopixel regime. It thus seems likely that bare absorber designs will lead the way in FIR/submm astronomical applications, above roughly 400-500 GHz, while antenna-coupled designs of one or another type, possible incorporating feedhorns, will dominate below $400 \mathrm{GHz}$ for CMB polarization applications. Sunyaev-Zeldovich effect mapping will likely continue to employ both kinds of techniques.

\section{OPTICAL COMPONENTS}

Though not necessarily strictly "low-temperature", optical components are inseparable from FIR/submm/mm detector design . Light at these wavelengths falls between the "optical" regime, in which one shapes the EM field by reflecting, focusing, and spatially terminating it, and the "microwave" regime, in which one directly manipulates the EM field, resulting in a mixture of these techniques. In this section, we discuss: thermal and bandpass spectral filtering, anti-reflection coating, spectral dispersion, and polarization analysis and modulation.

\section{Spectral Filtering}

Filtering and bandpass definition are generic to all astronomical instruments and provide an interesting example of the optical/microwave interface.

Very early experiments defined spectral bands using purely dielectric filters, whose absorption cutoffs would define broad, rather smooth-edged bands (e.g., [90]). But, because it is possible to make sub-wavelength structures easily by photolithography, the Cardiff group (then
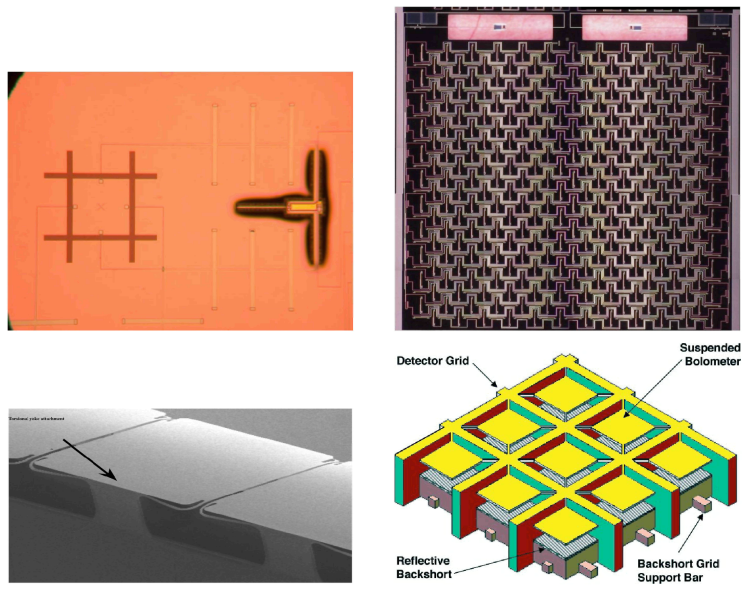

FIGURE 4. Array-format antenna-coupled and bareabsorber pixels. Top left: Berkeley dual slot dipole antenna with $\lambda / 4$ stub filters and TESs (A. Lee, Berkeley). Top right: dual-polarization phased-array antenna pixel with TESs at the right. Much of what is visible is the transmission line routing; the slot dipole antennae are the short vertical and horizontal lines. Bottom left: GSFC pop-up detector - the entire square supported on the torsional legs is the absorber, metallized to match free space. It would be implanted to make a semiconducting thermistor or have a TES deposited on it. Bottom right: waffle-pattern bare absorber schematic. GSFC figures courtesy of D. Benford.

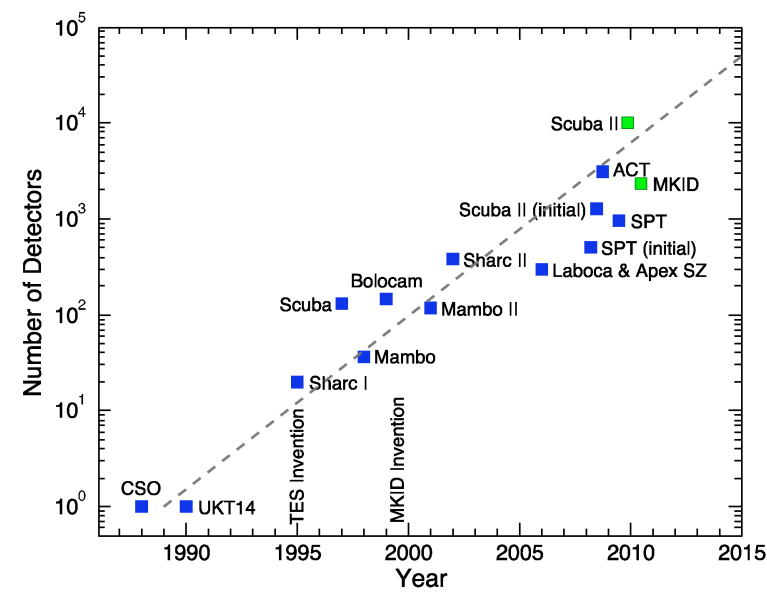

FIGURE 5. Bolometer array size as a function of time. Blue indicates deployed instruments, green indicates instruments under construction or proposed. Figure courtesy of J. Zmuidzinas [10].

at QMWC in London) began to construct optical filters using "inductive" and "capacitive" meshes [91] similar to lumped-element filters one uses in electrical circuits. Rather than being discrete components in a waveguide or electric circuit, they pattern these meshes onto lowloss dielectric films and place them directly in the op- 
tical path. Thinner, high-frequency variants of so-called "metal-mesh" filters can block thermal infrared radiation from $300 \mathrm{~K}$ and $77 \mathrm{~K}$ cryostat layers.

More recently, with the advent of superconducting transmission lines, one can use the microwave technique of placing lumped-element versions of such filters in transmission line to define spectral bandpasses, as noted earlier. A possible disadvantage is that fabrication variations set uniformity of response. On the other hand, metal-mesh filters display some variation of bandpass with angle of incidence and illumination. In-line bandpass filters are becoming the preference for antennacoupled submm $/ \mathrm{mm}$ architectures, while metal-mesh filters will remain in heavy use for absorber-coupled designs, especially in the FIR/short-submm.

Additionally, thermal infrared blocking - shielding one's cryogenic system and detectors against $300 \mathrm{~K}$ and $77 \mathrm{~K}$ radiation - must necessarily remain the province of optical filters because superconducting transmission lines provide no protection. Dielectric and metal-mesh filters [92] are in wide use for these purposes.

\section{Anti-reflection Coatings}

An element that is becoming critical but is so far not well-developed is wide-bandwidth anti-reflection (AR) coatings. Many architectures can sense a wide range of colors in a single focal plane, some even in a single pixel. But optical windows and lenses yield reflections due to the index mismatch to vacuum. A dielectric layer of thickness $\lambda / 4 \sqrt{n}$ consisting of a medium of index $\sqrt{n}$, where $n$ is the index of the dielectric being coated, is an ideal AR coating at one frequency and works well over $25 \%$ bandwidth (see, e.g., [93]). But polychromatic focal planes require more sophisticated coatings. A stack of layers can function well, but fabrication is challenging because the layers are macroscopically thick, unlike in the optical/near-infrared, where they can be deposited by sputtering or evaporation. Micromachining techniques make possible graded index AR coatings, where one adiabatically varies the material density, but they require extreme aspect ratios $(30: 1)$ for the graded structure [94, 95] and thus remain to be demonstrated. They would be especially challenging to fabricate on curved optical surfaces. Fortunately, as $n$ increases and AR coatings become more necessary, the corresponding optics require less curvature.

\section{Spectral Dispersion}

Another regime in which cryogenic optical elements play a significant role is dispersive spectroscopy. ZEUS
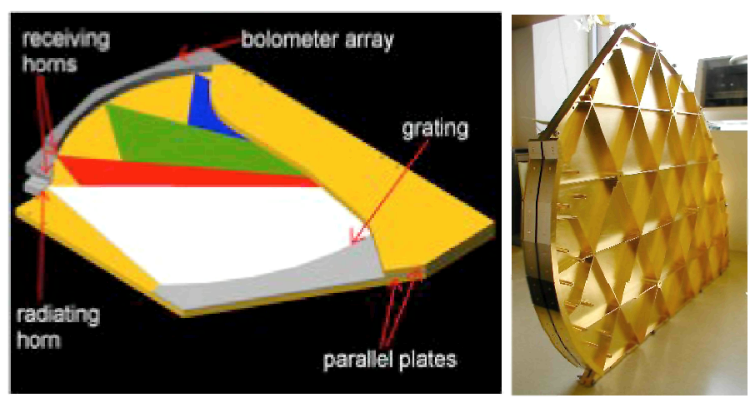

FIGURE 6. Planar Rowland grating design used in Z-Spec. The propagation plane is sandwiched between two parallel plates that define a waveguide structure. Figures courtesy of M. Bradford.

uses a classical optical Echelle grating design to provide spectral dispersion. Ruling such a grating is relatively easy given the large wavelength; however, the grating must be of order $R \lambda$ in size, resulting in rather large gratings for even moderate $R=1000$. Z-Spec offers the first hybridization of optical and microwave techniques [43]. Its design confines the grating to a parallel-plate waveguide structure, feeds it with light received by a feedhorn and coupled in via an emitting horn, disperses it by a grating at the edge of the waveguide medium, and receives the light at the detectors with a waveguide structure; see Figure 6. By confining the light in one dimension, the physical size in that dimension can be kept quite small and allows for stacking of multiple gratings, but the grating maintains a size $R \lambda$ in the other dimensions. Using a low-loss dielectric as the waveguide medium would shrink all dimensions by a factor $n ; n \approx 3.4$ for silicon makes this substantial. Some have proposed doing the dispersion step in a photolithographically fabricated planar waveguide structure, but this has not been implemented to date.

Higher-resolution spectroscopy in the $\mathrm{FIR} / \mathrm{submm} / \mathrm{mm}$ is usually done with SIS tunnel junction or hot-electron-bolometer mixers operating at $4 \mathrm{~K}$. The LTD conferences have historically not focused on these detectors, so we omit discussion of them here. They are always less sensitive than spectrometers using incoherent detectors, but they provide much higher resolving powers without the concomitant size increase.

\section{Polarization Analysis and Modulation}

A great deal of work has gone into the problem of polarization measurement in recent years because of the emphasis on CMB polarization anisotropy with its stringent requirements on sensitivity and systematics. Measuring percent-level polarization is a classic "differencing of two large numbers" problem: systematics that 


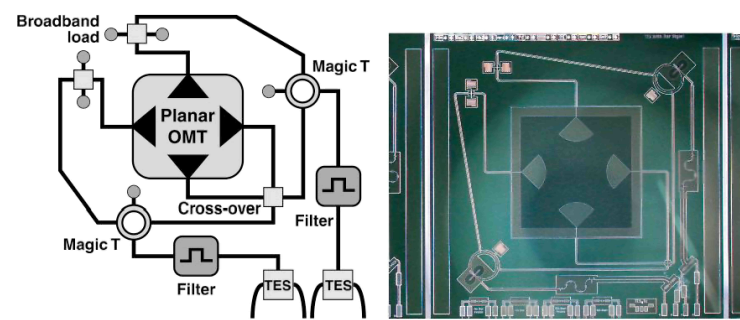

FIGURE 7. GSFC implementation of planar OMT. Left: Schematic design feeding two TES detectors via in-line bandpass filters. Right: Prototype device, mapping onto the schematic at left. Figure from [96].

might affect an intensity measurement at the percent level can dominate a polarization measurement. The two critical optical elements are a polarization analyzer to provide the two numbers to difference and a polarization modulator to control systematics by varying the polarization sensitivity with time in a controlled fashion.

As already described above, there are a wide number of polarization-sensitive feed/detector architectures for analyzing polarization. It is next necessary to modulate it. Modulation mitigates the "differencing two large numbers" problem: given two detectors that measure complementary polarizations, exchanging which polarization is sensed by which detector tests whether one is measuring a polarization on the sky rather than a difference in the calibrations of the two detectors responding to a much larger unpolarized signal. Additionally, to fully characterize the linear polarization on the sky, one needs to measure the difference of signal in the two polarizations in two independent orientations of the pair rotated by $45^{\circ}$ relative to one another, known as $Q$ and $U$. If one uses two pairs of detectors rotated relative to one another, the same relative calibration problem enters in comparing $Q$ and $U$. It is thus desirable to rotate the polarization sensitivity angle of a single pair of detectors on a short timescale. Finally, modulating the polarization at a frequency above the $1 / f$ noise of the detector renders the measurement immune to that noise.

There are a number of polarization modulators in development. An extensive review is given in [96]. The simplest and most historically well-studied is the halfwave plate (HWP) made of a birefringent material such as sapphire. A HWP introduces a phase shift of $180^{\circ}$ between two monochromatic electromagnetic waves that have orthogonal polarizations as they travel through it. It thereby rotates the incoming polarization by an angle $2 \theta$ where $\theta$ is the angle between the incoming polarization and the "fast" axis of the HWP. Thus, a specific set of angles rotate the polarization axes by $90^{\circ}$, providing the first kind of modulation described above (also known as $Q \rightarrow-Q$ ). Another specific set of angles rotate the polarization axes by $45^{\circ}$, or $Q \rightarrow U$. And, finally, one would like to step or rotate the HWP quickly compared to the $1 / f$ noise. In principle, the HWP rotates polarization ( $\vec{E}$ field direction) without rotating the beam ( $\vec{E}$ field magnitude), thus avoiding spurious signals in the above rotations due to an azimuthally beam rotating on the sky.

There are a number of practical challenges, however. First, the HWP polarization rotation angle changes with frequency because the HWP condition only holds at one frequency. This can be addressed with a stack of HWPs each designed for a different frequency, yielding a HWP that is approximately achromatic across an octave spectral band. Second, the HWP suffers the generic AR-coat problem explained earlier. There are two published achromatic waveplates that largely solve these problems $[97,98,99]$. Second, because of the index difference between the fast and slow axes, transmission and reflection will be mildly angle-dependent, though it is expected that this is modulated at half the rotation period of the polarization itself. Finally, because the HWP is used in transmission, loss and loading are not negligible and cryogenic operation is desirable, a point that will discussed below. A number of experiments plan sapphire HWPs, including EBEX, BICEP2/Keck Array, SPIDER, ABS (warm), POLARBeaR, ACTPol, SPTPol, and, formerly, $\mathrm{C}_{\ell}$ OVER. Most will use a monochromatic version, with a single sapphire plate with a single $A R$ layer to obtain $\sim 25 \%$ bandwidth, but EBEX requires the achromatic version and a number of the others will likely upgrade when available.

The cryogenic operation of the HWP is a significant challenge. Ideally, one would rotate the HWP much faster than the beam-crossing time on the sky so that all the aforementioned problems are optimally addressed. One thus needs low-dissipation bearings and drives. A superconducting magnetic bearing has been developed [100] as well as an induction drive [101]. EBEX used the dissipationless bearing with a belt connected to a shaft driven by a warm motor in its test flight. All the other experiments currently approaching deployment - BICEP2/Keck, SPIDER, and POLARBeaR - will step the waveplate intermittently, allowing them to use contacting bearings and dissipative drives. They are then more subject to $1 / f$ noise and calibration drift.

Sapphire HWPs are limited by the accessibility of sapphire in sufficiently large size at reasonable cost. The largest diameter currently available is $30 \mathrm{~cm}$. The Cardiff group has begun to develop a HWP based on its wellknown metal-mesh filter technology [96]. The necessary phase delay is provided by anisotropic capacitive and inductive mesh filters, which also serve to define the bandpass of the HWP. Lab tests of $3.5-\mathrm{cm}$ diameter prototypes have confirmed calculations. Deployment in instruments awaits development of larger versions. 

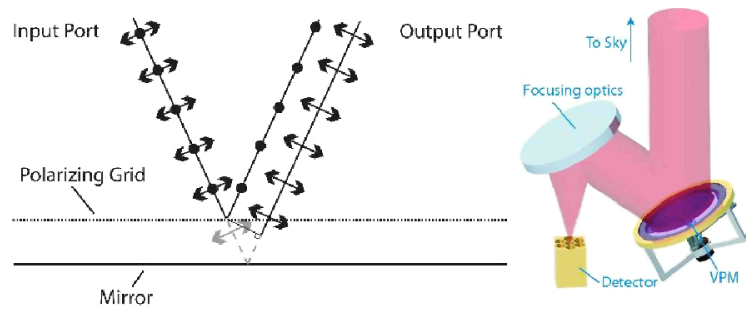

FIGURE 8. Principle of operation of the variable-delay polarization modulator. The wire grid reflects the polarization perpendicular to the page, while the mirror reflects the other polarization. A phase delay is created by the additional path length for the polarization in the plane of the page. Left figure from [102], right figure from [96].

The variable-delay polarization modulator (VPM) [102] is also an optical technique using reflection instead of transmission. By placing a polarizing wire grid in front of a reflective surface, one creates a path-length delay between the two polarizations; see Figure 8 . One modulates the delay by translating the reflector along the line between the reflector and the grid. Clearly, it is an elegantly simple technique, but it does suffer some disadvantages: it only measures $Q$, the beams of the two polarizations are displaced by the reflection because the beam always must make an angle to the normal, and this displacement is synchronous with the delay modulation; the grid and mirror must be kept parallel; and, because the mirror is being translated, the speed of modulation is limited. Initial tests have demonstrated basic functionality [102].

Two other techniques modulate polarization in transmission line after reception using MEMS or SIS-tunneljunction devices to switch which polarization goes to which detector, yielding the $Q$ signal at the switch frequency and the mean intensity at zero frequency [96]. One can create phase lags using transmission-line hybrids to convert $Q$ to $U$ in a manner similar to the way a half-wave plate works. Both elements can be made reasonably broadband $(25 \%)$ to function over a single spectral band. These devices offer the ultimate "polarimeteron-a-chip." However, they are not yet the obvious choice because, ideally, one modulates polarization at the input of the optical chain rather than the end: All optical elements display some polarization-dependent behavior and thus a focal-plane polarization modulator will create a spurious signal. Thus, many first-generation polarization experiments are using HWPs or VPMs. It may turn out that, as with many other similar fears in the past, the systematic signal created by a focal-plane modulator is very stable and easily removed, so such designs are certainly worth pursuing to the point of field demonstration.

\section{CONCLUSION AND OUTLOOK}

We have summarized a wide variety of ongoing developments in low-temperature detectors for cosmic microwave background work and submm astronomy. Background-limited performance in progressively more demanding regimes has been achieved by a number of technologies and continues to march progressively downward in NEP. Kilopixel array formats of a wide range of designs have been demonstrated and larger pixel counts are in the works. A number of optical design issues are strongly coupled to detector design, and some optical components are now moving into the focal plane itself as they are shifted from the optical regime to the microwave regime. We look forward to continued progress on all these fronts.

\section{ACKNOWLEDGMENTS}

We thank all those who have provided information used in this paper. This work was supported by the California Institute of Technology and the Gordon and Betty Moore Foundation.

\section{REFERENCES}

1. C. L. Bennett et al., Astroph. J. Lett. 464, L1 (1996).

2. J.-L. Puget et al., Astron. Astroph. 308, L5 (1996).

3. M. G. Hauser et al., Astroph. J. 508, 25 (1998).

4. D. J. Fixsen et al., Astroph. J. 508, 123 (1998).

5. D. J. Schlegel et al., Astroph. J. XXX (1998).

6. I. Smail et al., Astroph. J. Lett. 490, L5 (1997).

7. A. W. Blain et al., Astroph. J. Lett. 512, L87 (1999).

8. H. Dole et al., Astron. Astroph. 451, 417 (2006).

9. K. Irwin, this volume.

10. J. Zmuidzinas, in prep. for Annu. Rev. Astro. Astroph.

11. J. J. Bock et al., Sp. Sc. Rev. 74, 229 (1995).

12. P. D. Mauskopf et al., Appl. Opt. 36, 765 (1997).

13. B. P. Crill et al., Astroph. J. Suppl. 148, 527 (2003).

14. J. F. Macías-Pérez et al., Astron. Astroph. 467, 1313 (2007).

15. http://sci.esa.int/science$\mathrm{e} / \mathrm{www} / \mathrm{area} / \mathrm{index} . \mathrm{cfm}$ ?fareaid $=17$.

16. M. Griffin et al., in Proceedings of the SPIE (SPIE, Bellingham, Washington, 2008), Vol. 7020.

17. C. D. Dowell et al., in Proceedings of the SPIE (SPIE, Bellingham, Washington, 2003), Vol. 4855, pp. 73-87.

18. N. Billot et al., in Proceedings of the SPIE (SPIE, Bellingham, Washington, 2006), Vol. 6265.

19. P. de Bernardis et al., Nature 404, 955 (2000).

20. S. Hanany et al., Astroph. J. 545, L5 (2000).

21. A. Benoit et al., Astron. Astroph. 399, L25 (2003).

22. C. L. Reichardt et al., Astroph. J. 694, 1200 (2009).

23. G. T. Laurent et al., Astroph. J. 643, 38 (2006).

24. A. Kovács et al., Astroph. J. 650, 592 (2006).

25. M. Devlin et al., Nature 458, 737 (2009).

26. M. Kenyon et al., this volume. 
27. A. T. Lee et al., Appl. Phys. Lett. 69, 1801 (1996).

28. S. Lee et al., Appl. Opt. 37, 3391 (1998).

29. J. M. Gildemeister et al., Appl. Phys. Lett. 74, 868 (1999).

30. J. Mehl et al., J. Low. Temp. Phys. 151, 697 (2008).

31. D. Swetz et al., in Proceedings of the SPIE (SPIE, Bellingham, Washington, 2008), Vol. 7020.

32. Y. Zhao et al., in Proceedings of the SPIE (SPIE, Bellingham, Washington, 2008), Vol. 7020.

33. A. Orlando et al., this volume.

34. J. Austermann et al., this volume.

35. T. Stevenson et al., J. Low. Temp. Phys. 151, 471 (2008).

36. P. K. Day et al., Nature 425, 817 (2003).

37. P. R. Maloney et al., The MKID Camera this volume.

38. J. Baselmans et al., this volume.

39. J. Schlaerth et al., this volume.

40. B. Mazin, this volume

41. A. Benoit et al., in Proceedings of the SPIE (SPIE, Bellingham, Washington, 2008), Vol. 7020.

42. M. Piat et al., in Proceedings of the SPIE (SPIE, Bellingham, Washington, 2008), Vol. 7020.

43. L. Earle et al., in Proceedings of the SPIE (SPIE, Bellingham, Washington, 2006), Vol. 6275, pp. $627510+$.

44. G. J. Stacey et al., in Proceedings of the SPIE (SPIE, Bellingham, Washington, 2004), Vol. 5498, pp. 232-245.

45. B. Naylor, Ph.D. thesis, California Institute of Technology, 2007.

46. L. Earle, Ph.D. thesis, University of Colorado, Boulder, 2008.

47. S. Hailey-Dunsheath et al., Astroph. J. Lett. 689, L109 (2008).

48. S. Hailey-Dunsheath et al., in EAS Publications Series (EAS, France, 2008), Vol. 31, pp. 159-162.

49. P. Schloerb, in Proceedings of the SPIE (SPIE, Bellingham, Washington, 2008), Vol. 7012.

50. T. Sebring et al., in Proceedings of the SPIE (SPIE, Bellingham, Washington, 2008), Vol. 7012.

51. T. Nakagawa, in Proceedings of the SPIE (SPIE, Bellingham, Washington, 2008), Vol. 7010.

52. D. F. Lester et al., in Proceedings of the SPIE (SPIE, Bellingham, Washington, 2004), Vol. 5487, pp. 1507-1521.

53. P. F. Goldsmith et al., in Proceedings of the SPIE (SPIE, Bellingham, Washington, 2008), Vol. 7010.

54. B. Karasik et al., in Proceedings of the SPIE (SPIE, Bellingham, Washington, 2008), Vol. 7020.

55. B. Karasik et al., this volume.

56. E. M. Barrentine et al., J. Low. Temp. Phys. 151, 173 (2008).

57. E. M. Barrentine et al., this volume.

58. P. Mauskopf et al., in Proceedings of the SPIE (SPIE, Bellingham, Washington, 2008), Vol. 7020.

59. S. Doyle et al., in Proceedings of the SPIE (SPIE, Bellingham, Washington, 2008), Vol. 7020.

60. J. Baselmans et al., J. Low. Temp. Phys. 151, 524 (2008)

61. R. Barends et al., J. Low. Temp. Phys. 151, 518 (2008).

62. W. C. Jones et al., in Proceedings of the SPIE (SPIE, Bellingham, Washington, 2003), Vol. 4855, pp. 227 -.

63. J. Glenn et al., Appl. Opt. 41, 136 (2002).

64. D. J. Haig et al., in Proceedings of the SPIE, Vol. 5498 : Millimeter and Submillimeter Detectors for Astronomy II, edited by J. Zmuidzinas and W. S. Holland (SPIE,
Bellingham, Washington, 2004), pp. 78-94.

65. E. Kreysa et al., in Proceedings of the SPIE (SPIE, Bellingham, Washington, 1998), Vol. 3357, pp. 319-325.

66. G. Siringo et al., in Proceedings of the SPIE (SPIE, Bellingham, Washington, 2008), Vol. 7020.

67. E. Pascale et al., Astroph. J. 681, 400 (2008).

68. W. Grainger et al., in Proceedings of the SPIE (SPIE, Bellingham, Washington, 2008), Vol. 7020.

69. J. Britton et al., this volume.

70. R. W. Haas et al., ijirmmw 14, 2289 (1993).

71. A. Crites et al., this volume.

72. A. Vayonakis et al., AIP Conf. Proc. 605: Low Temperature Detectors 605, 539 (2002).

73. S. Kumar et al., IEEE Trans. Appl. Supercon. 19, 924 (2009).

74. A. Goldin et al., in Proceedings of the SPIE, Vol. 4855: Millimeter and Submillimeter Detectors for Astronomy, edited by T. G. Phillips and J. Zmuidzinas (SPIE, Bellingham, Washington, 2003), pp. 163-171.

75. M. Myers et al., J. Low. Temp. Phys. 151, 464 (2008).

76. R. O'Brient et al., J. Low. Temp. Phys. 151, 459 (2008).

77. R. O'Brient et al., this volume.

78. L. E. Bleem et al., this volume.

79. K. Denis et al., this volume.

80. J. McMahon et al., this volume.

81. L. Piccirillo et al., in Proceedings of the SPIE (SPIE, Bellingham, Washington, 2008), Vol. 7020.

82. J. Zmuidzinas and H. G. LeDuc, IEEE Trans. Micro. Th. Tech. 40, 1797 (1992).

83. G. Chattopadhyay and J. Zmuidzinas, IEEE Trans. Ant. Prop. 46, 736 (1998).

84. C. L. Kuo et al., in Proceedings of the SPIE (SPIE, Bellingham, Washington, 2008), Vol. 7020.

85. D. J. Benford et al., in Proceedings of the SPIE (SPIE, Bellingham, Washington, 2004), Vol. 5498, pp. 647-658.

86. J. Staguhn et al., in Proceedings of the SPIE (SPIE, Bellingham, Washington, 2008), Vol. 7020.

87. S. Dicker et al., in Proceedings of the SPIE (SPIE, Bellingham, Washington, 2008), Vol. 7020.

88. M. D. Audley et al., in Proceedings of the SPIE (SPIE, Bellingham, Washington, 2008), Vol. 5498.

89. V. Revéret et al., J. Low. Temp. Phys. 151, 32 (2008).

90. M. Halpern et al., Appl. Opt. 25, 565 (1986).

91. P. A. R. Ade et al., in Proceedings of the SPIE (SPIE, Bellingham, Washington, 2006), Vol. 6275.

92. C. E. Tucker and P. A. R. Ade, in Proceedings of the SPIE (SPIE, Bellingham, Washington, 2006), Vol. 6275.

93. C. L. Kuo, J. Phys. C 155, 012007/10 (2009).

94. C. L. Kuo, private communication.

95. J. McMahon, J. Phys. C 155, 012007/10 (2009).

96. P. A. R. Ade et al., J. Phys. C 155, 012006/1 (2009).

97. S. Hanany et al., Appl. Opt. 44, 4666 (2005).

98. G. Savini et al., Appl. Opt. 45, 8907 (2006).

99. G. Pisano et al., Appl. Opt. 45, 6982 (2006).

100. S. Hanany et al., IEEE Trans. Appl. Supercon. 13, 2128 (2003).

101. T. Matsumura et al., Physica C Superconductivity 426, 746 (2005).

102. M. Krejny et al., Appl. Opt. 47, 4429/1 (2008). 